\title{
Simulation and Analysis on Average Bond Lengths in GalnNAs Electrical Materials
}

\author{
Tang Lili ${ }^{* 1, a}$, Huang Wei ${ }^{2, b}$ \\ ${ }^{1}$ Department of Computer \& Information Engineering, Zhixing College of Hubei University, Wuhan, \\ 430011, China \\ ${ }^{2}$ System Design Institute of Hubei Aerospace Technology Academy, Wuhan, 430040, China \\ aemail: toney2001@126.com, bemail:jijunhuang@126.com
}

\section{Keyword: Simulated Calculation; GalnNAs; Average Bond Length}

\begin{abstract}
This article describes the bond length distribution of the Ga1-xlnxNyAs1-y quaternary alloy which has attracted much attention in recent years for its potential use in fabricating long wavelength optoelectronic devices, and propose a statistical model, which calculates the average bond lengths in as-grown and fully annealed Ga1-xlnxNyAs1-y alloys when constituent proportion is an integer and $x: y \geq 4$. First we calculate the statistical bond distributions of a definite super cell, and then generalize the formulas of the average lengths after extracting the distributing probabilities of the $\mathrm{N}$-centered nearest-neighbor clusters with a $\sigma^{2}=1$ Gaussian distribution based on the former experiment. Finally the optimized bond lengths of Ga28ln4N1As31 super cells under different nearest-neighbor environments are obtained using DMol3 module in Material Studio based on the first principle, and the average bond lengths and strain are computed. We find that the local strain, the total strain and total binding energy of the system will decrease after annealing, which is in good agreement with the previous researches.
\end{abstract}

\section{Introduction}

The $\mathrm{Ga}_{1-\mathrm{x}} \ln _{\mathrm{x}} \mathrm{N}_{\mathrm{y}} \mathrm{As}_{1-\mathrm{y}}$ quaternary alloy has attracted much attention in recent years for its potential use in fabricating long wavelength optoelectronic devices [1]. As is well known, inserting an element In conventional III-V semiconductors such as GaAs or InP usually leads to a reduction in band gap energy and at the same time an increase in the lattice constant. But when $\mathrm{N}$ is inserted, the lattice constant will reduce as well as band gap energy. Accordingly, lattice matched $\mathrm{Ga}_{1-\mathrm{x}} \mathrm{ln}_{\mathrm{x}} \mathrm{N}_{\mathrm{y}} \mathrm{As}_{1-\mathrm{y}} / \mathrm{GaAs} \mathrm{QW}$ emitting at definite long wavelength can be achieved. However, the growth of this dilute nitride semiconductor is complicated by phase separation effects due to the solubility limit of nitrogen, which leads to poor as-grown photoluminescence (PL) efficiencies. Rapid thermal annealing (RTA) is usually used to decrease the density of the non-radioactive recombination centers and improve the material quality. Meanwhile, it causes a drastic blue shift of band gap [2][3]. A large number of spectra studies suggest that the nearest-neighbor atoms changes lead to blue shift [4]. Later studies have also confirmed that annealing makes the In-N bond lengths greatly increase, and can reduce total strain of the system [2][4]. But the exact ratio between In-N bond and other binary compounds has not been reported. We simulate $G_{28} \ln _{4} N_{1} A s_{31}$ super cells under different nearest-neighbor environments using $\mathrm{DMol}^{3}$ module in Material Studio based on the first principle, and discuss the strain before and after annealing.

\section{Theoretical Model}

In conventional ternary alloys, the proportion of different bonds is fixed. Such as, the ratio between In-As bond and Ga-As bond is $\mathrm{x}:(1-\mathrm{x})$ in $\mathrm{Ga}_{1-\mathrm{x}} \ln _{\mathrm{x}}$ As ternary alloys. But, in as-grown $\mathrm{Ga}_{1-\mathrm{x}} \ln _{\mathrm{x}} \mathrm{N}_{\mathrm{y}} \mathrm{As}_{1-\mathrm{y}}$ quaternary alloys, the proportion of the four different binary bonds is uncertain. Their distributions in all probability vary from Vegard's law and the deviation can be described using a "short range order" parameter $\xi$ [5], which is defined as

$$
\xi=n_{I n-N} / M-x_{I n} y_{N}
$$


Where $n_{I n-N}$ is the number of the nearest-neighbor (NN) In-N bonds, and M is the total number of the whole bonds.

It's certain that the number of Ga-N bonds is far more than In-N bonds if the binary bonds of $\mathrm{Ga}_{1-\mathrm{x}} \ln _{\mathrm{x}} \mathrm{N}_{\mathrm{y}} \mathrm{As}$ 1-y $_{\text {y }}$ alloys are completely random arranged ( $\xi=0$ ) [4].

Here we propose an assumption that the phase separation result of equimolar system GalnNAs at $\mathrm{T}=0 \mathrm{~K}$ is "Ga-N + In-As", in which constituents are incoherent with the substrate (GaAs). In other words, the configuration under assumed $0 \mathrm{~K}$ growth condition is determined only by less bond energy, and strain has little effect on it [3].

when the material is fully annealed, the distributions will be static and at definite annealing temperature $\mathrm{T}, \xi(x, y, T)$ is exclusive. So the proportions of each binary bond can be written as

$$
\begin{array}{ll}
\text { In-N: } & x y+\xi \\
\text { Ga-N: } & y(1-x)-\xi \\
\text { In-As: } & x(1-y)-\xi \\
\text { Ga-As: } & (1-x)(1-y)+\xi
\end{array}
$$

This $\xi(x, y, T)$ equation is easily positive solution and the number of $\mathrm{NN}$ In atoms per $\mathrm{N}$ is calculated and designated as $r$ parameter

$$
r=4(x y+\xi) / y
$$

In the ideal model, when constituent proportion is integer and $x: y \geq 4, G a_{1-x} \ln _{x} N_{y} A s_{1-y}$ alloys can be approximately divided into five $\mathrm{N}$-centered $\mathrm{NN}$ clusters $\mathrm{N}-\mathrm{In}_{\mathrm{s}} \mathrm{Ga}_{4-\mathrm{s}}$ and each super-unit cell are consistent ratio between components. So, the distributions probability of super-unit cell is determined entirely by clusters.

$\mathrm{Ga}_{1-\mathrm{x}} \ln _{\mathrm{x}} \mathrm{N}_{\mathrm{y}} \mathrm{As}_{1-\mathrm{y}}$ meeting the above composition conditions might as well be written in the form of super-unit cell $\mathrm{Ga}_{\mathrm{m}} \mathrm{ln}_{\mathrm{n}} \mathrm{NAs} \mathrm{s}_{\mathrm{m}+\mathrm{n}-1}$, so

$$
x=\frac{n}{m+n}, y=\frac{1}{m+n} \quad(\mathrm{~m}, \mathrm{n} \text { is an integer })
$$

We suppose that the five N-centered NN clusters $N$ - $\operatorname{In}_{s} \mathrm{Ga}_{4-\mathrm{s}}(\mathrm{s}=0,1,2,3,4)$ are homogeneously embedded in InGaAs, and with respect to $\mathrm{M}_{0}$ mol Ga ${ }_{1-\mathrm{x}} \ln _{\mathrm{x}} \mathrm{N}_{\mathrm{y}} \mathrm{As}_{1-\mathrm{y}}$ the number of each clusters are $k_{s}$ mol, respectively, so

$$
\begin{aligned}
& k_{0}+k_{1}+k_{2}+k_{3}+k_{4}=M_{0} y \\
& k_{1}+2 k_{2}+3 k_{3}+4 k_{4}=4 M_{0}(x y+\xi) \\
& 4 k_{0}+3 k_{1}+2 k_{2}+k_{3}=4 M_{0}[y(1-x)-\xi] \\
& (4 m-4) k_{0}+(4 m-3) k_{1}+(4 m-2) k_{2}+(4 m-1) k_{3}+4 m k_{4}=4 M_{0}[(1-x)(1-y)+\xi](11) \\
& 4 n k_{0}+(4 n-1) k_{1}+(4 n-2) k_{2}+(4 n-3) k_{3}+(4 n-4) k_{4}=4 M_{0}[x(1-y)-\xi]
\end{aligned}
$$

With a particular composition and annealing temperature, the corresponding $k_{s}$ can not be obtained from formula (8)-(12). Lordi et al [3] found that in as-growth $\mathrm{Ga}_{1-\mathrm{x}} \ln _{\mathrm{x}} \mathrm{N}_{\mathrm{y}} \mathrm{As}_{\text {1-y }}$ quaternary alloys $\mathrm{N}$ is around the center of the average number of $\mathrm{In}$ atoms, $\mathrm{N}-\mathrm{In}_{\mathrm{s}} \mathrm{Ga}_{4-\mathrm{s}}$ clusters are with a $\sigma^{2}=1$ Gaussian distribution. When achieving annealed thermal equilibrium, the conversion probability between the clusters is equal, also expected to meet the above distribution.

In this paper, the probability distribution of five clusters is $\mathrm{K}_{\mathrm{s}} / 4 \mathrm{M}_{0}$. May wish to make it meet the r-centered Gaussian distribution, then the probability distribution of the binary compounds in five different super cells is as following equation.

$$
p_{s}=\frac{1}{\sqrt{2 \pi} \sigma} \exp \left[(s-r)^{2} / 2 \sigma^{2}\right]
$$

In this paper, the average bond length of In-N bond can be written as 


$$
\bar{d}_{I n-N}=\frac{\sum_{s=0}^{4} s * p_{s} * d_{I n-N}^{s}}{\sum_{s=0}^{4} s * p_{s}}
$$

Where $d_{I n-N}^{s}$ is the In-N bond in the corresponding super cell, $\mathrm{s}$ is the number of In-N bond.

For the Ga-As bond, we sum up the formula of the average bond length as follows:

$$
\bar{d}_{G a-A s}=\frac{\sum_{s=0}^{4}(4 m-4+s) * p_{s} * d_{G a-A s}^{s}}{\sum_{s=0}^{4}(4 m-4+s) * p_{s}}
$$

Similarly, we get the average bond lengths of Ga-N bond and the In-As bond.

Considering the strain of the binary alloy, the strain can be obtained from the changes of the average bonds. So the revised average bond lengths of the strain contained binary components can be written as:

$$
\bar{d}_{i-j}=\left(1+\varepsilon_{i-j}\right) * d_{i-j}
$$

Where $\mathrm{i}$ is cation and $\mathrm{j}$ is anion, $\mathrm{d}$ is bond length of the corresponding intrinsic compounds.

\section{Modeling and simulated calculation}

The ideal GaAs is blende structure, belonging to $\mathrm{F} 43 \mathrm{~m}$ space group, with $\mathrm{a}=\mathrm{b}=\mathrm{c}=0.565315 \mathrm{~nm}$, $\alpha=\beta=\gamma=90^{\circ}$. We simulate the structure and physical characteristics of $G_{28} \ln _{4} N_{1} A s_{31}$ super cells under different nearest-neighbor environments using $\mathrm{DMol}^{3}$ module based on the first principle. Considering III - V elements of small clusters close to the ground state structure, in establishing $\mathrm{Ga}_{1-\mathrm{x}} \mathrm{In}_{\mathrm{x}}$ As model, GaAs is first used as the primary structure of super cell, with the atom As in the very centre. Then the atom As is substituted by atom $\mathrm{N}$, and N-nearest neighbor In atoms increase from 0 to 4 in number, and atom In is to be distributed in super cell symmetrically.

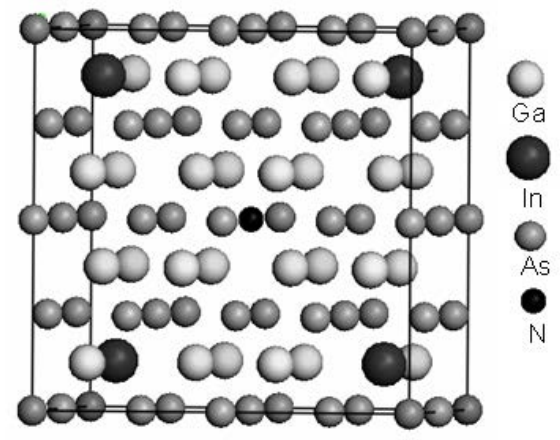

Fig.1. Blende structure $\mathrm{Ga}_{28} \ln _{4} \mathrm{~N}_{1} \mathrm{As}_{31}$ super cells with 64 atoms

In figures 1, we simulate the structure and physical characteristics of blende structure $\mathrm{Ga}_{28} \ln _{4} \mathrm{~N}_{1} \mathrm{As}_{31}$ super cells with 64 atoms under different nearest-neighbor environments, and get the corresponding bond length and the total binding energy when $\mathrm{N}$-nearest neighbor In atoms increase from 0 to 4 in number, the binding energy of five super cells, respectively, are -265.32622 $\mathrm{eV},-265.55357 \mathrm{eV},-265.63017 \mathrm{eV},-265.87206 \mathrm{eV}$ and $-265.95466 \mathrm{eV}$. The total binding energy of the system will decrease after annealing, which is in good agreement with the increase in the number of In-N bonds.

\section{Results and Discussion}

Figure 2 shows the relationship between $\mathrm{r}$ and the probability distribution $\mathrm{P}_{\mathrm{s}}$ of five kind clusters in $\mathrm{Ga}_{28} \ln _{4} \mathrm{~N}_{1} \mathrm{As}_{31}$ cell. We can see directly, N-nearest neighbor clusters dominated by $\mathrm{N}-\mathrm{In}_{0} \mathrm{Ga}_{4}$ and $\mathrm{N}-\mathrm{In}_{1} \mathrm{Ga}_{3}$ in as-growth alloy; After the fully annealed with $\mathrm{T}=800 \mathrm{~K}$, In-N bonds significantly increased in number, and $\mathrm{N}$-nearest neighbor clusters dominated by $\mathrm{N}-\mathrm{In}_{2} \mathrm{Ga}_{2}$ with increasing 
annealing temperature. When the component ratio x:y is much larger than 4, corresponding peak of Guassion distribution will be close to 3 .

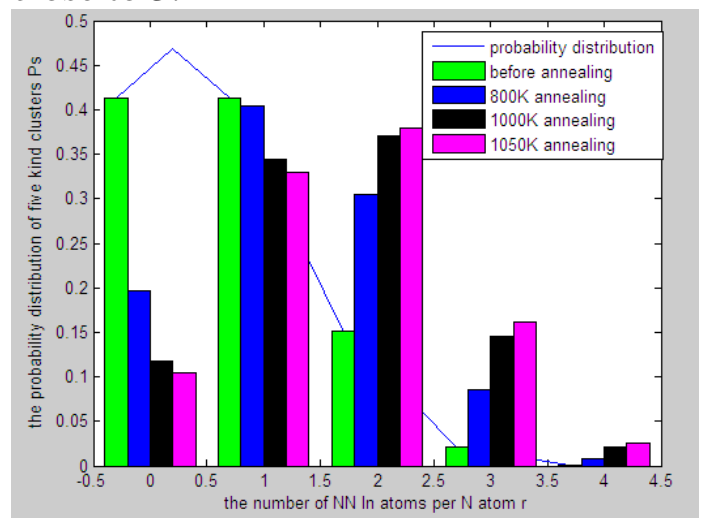

Fig.2. The relation between the probability distribution $\mathrm{P}_{\mathrm{s}}$ of five kind clusters and $\mathrm{r}$ Tab.1. shows the average bond length of four compounds in the strain-free, as-growth and complete annealed

\begin{tabular}{c|c|c|c|c|c}
\hline $\begin{array}{c}\text { binary } \\
\text { compounds }\end{array}$ & $\begin{array}{c}\text { strain-free } \\
\bar{d}_{0} / \mathrm{nm}\end{array}$ & $\begin{array}{c}\text { as-growth } \\
\bar{d} / \mathrm{nm}\end{array}$ & $\begin{array}{c}\text { complete } \\
\text { annealed } \\
\bar{d}_{\text {ann. }} / \mathrm{nm}\end{array}$ & $\begin{array}{c}\text { as-growth } \\
\text { strain (\%) }\end{array}$ & $\begin{array}{c}\text { annealed } \\
\text { strain (\%) }\end{array}$ \\
\hline In-N bond & 0.21400 & 0.22365 & 0.22035 & 4.51 & 2.97 \\
\hline Ga-N bond & 0.19500 & 0.20371 & 0.20097 & 4.47 & 3.06 \\
\hline In-As bond & 0.26200 & 0.25965 & 0.26012 & -0.9 & -0.72 \\
\hline Ga-As bond & 0.24500 & 0.24450 & 0.24439 & -0.2 & -0.25 \\
\hline
\end{tabular}

Table 1 shows the average bond length of four compounds in the strain-free, as-growth and complete annealed $(\mathrm{T}=800 \mathrm{~K})$ conditions. We can see from the data in the table, the average bond length of In-N bond and Ga-N bond reduce. From formula (16), we calculated annealed strain is about $3 \%$, and the strain reduction caused by annealing about $1.5 \%$. The strain in In-As bond decreased slightly, while increased slightly in Ga-As bond.

\section{Conclusions}

In this thesis, we simulate and obtain the optimized bond lengths, and sum up the average bond lengths. The results show that the local strain reduce in annealing and reduce by $3 \%$ after complete annealing, and simultaneously the total strain and total binding energy of the system will decrease after annealing, which is in good agreement with the previous researches. The above theoretic simulation on physical parameters is also of practical significance and is expected to offer some reference to researchers and industrial circles in designing $\mathrm{Ga}_{1-\mathrm{x}} \ln _{\mathrm{x}} \mathrm{N}_{\mathrm{y}} \mathrm{As}_{1-\mathrm{y}}$ based semiconductor components.

\section{References}

[1] Kondow M, Uomi K, Niwa A, et al. GaInNAs: a novel material for long-wavelength -range laser diodes with excellent high temperature performance[J]. Jpn. J Appl Phys, 1996, 35:1273-1275

[2] Kondow M, Kitatani T and Shirakata S. Annealing in GaInNAs system [J]. J Phys: Condens Matter, 2004, 16:S3229

[3] Lordi V, Yuen H B, Bank S R, et al. Nearest-neighbor distributions in $\mathrm{Ga}_{1-\mathrm{x}} \ln _{\mathrm{x}} \mathrm{N}_{\mathrm{y}} \mathrm{As} \mathrm{s}_{1-\mathrm{y}}$ and $\mathrm{Ga}_{1-\mathrm{x}} \ln _{\mathrm{x}} \mathrm{N}_{\mathrm{y}} \mathrm{As}_{1-\mathrm{y}-\mathrm{z}} \mathrm{Sb}_{\mathrm{z}}$ thin films upon annealing[J]. Phys Rev B, 2005, 71(12):125309

[4] Lordi V, Gambin V, Friedrich S, et al. Nearest-Neighbor Configuration in (GaIn)(NAs) Probed by X-Ray Absorption Spectroscopy[J]. Phys Rev Lett, 2003, 90(14):145505

[5] Kim K and Zunger A. Spatial Correlations in GaInAsN Alloys and their Effects on Band-Gap Enhancement and Electron Localization [J]. Phys Rev Lett, 2001, 86(12):2609 